\title{
Research on Construction and Implementation Scheme of Wireless Network in Universities
}

\author{
Wang Kefei ${ }^{1, a}$, Lu Ming ${ }^{1, b}$ and Ke Hongdi ${ }^{1, ~}{ }^{*} \mathrm{c}$ \\ 1 Department of Technology,Jilin Business and Technology College,Changchun 130507, China

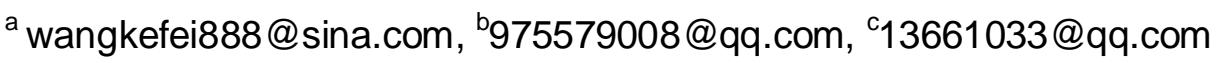

Keywords: Wireless Network; WLAN; AP; RTSG

\begin{abstract}
This paper takes the wireless network construction project of Jilin Business and Technology College as the research object, this paper introduces the general principles and technical requirements of the construction of Wireless campus network, constructs the network topology based on the above principles, designs the implementation plan of the wireless network of Jilin Institute of Commerce and Industry, launches the implementation construction according to the design plan, and finally analyzes the advantages of the implementation plan.
\end{abstract}

\section{Introduction}

With the development of university informatization, the change of the content in the network and the maturity of the new access mode.The requirements of modern WLAN wireless campus network are summarized as follows: Wireless coverage, compatible with wired network, security requirements, stable and reliable, certified billing, operation and maintenance.

The construction and implementation of the new campus network of Jilin Business and Technology College in the same way, according to the actual situation of the new campus of Jilin Business and Technology College, this paper designs the implementation plan of the wireless network of Jilin Industrial and Commercial College, according to the design plan.

\section{Main Technology}

In the Wireless campus network solution, the core switch $+\mathrm{AC}$ controller is adopted to realize the unified management of all indoor and outdoor AP in the Wireless campus network in this period.

In the case of the constant number of devices, the access capacity of the network is increased by one times to meet the rapid growth of WLAN users; Both indoor and outdoor AP use dual-frequency mode, that is, can work at the same time in the $2.4 \mathrm{GHz}$ and $5 \mathrm{GHz}$ band.Outdoor AP using a professional outdoor thin AP, fully considering the complexity of outdoor application environment, must be able to meet the waterproof, dustproof, lightning protection, high and low temperature and other requirements under a variety of environments. At the same time can accept the unified management of wireless controllers.

PoE switch with all gigabit access to the million Gigabit uplink PoE switch, according to the AP deployment, the largest switch to provide more than $370 \mathrm{~W}$ power supply, to ensure the line-speed all gigabit forwarding, achieve gigabit to the terminal.Management, the wireless controller, indoor AP and Outdoor AP equipment are managed by adding wireless network system on the NMS.

Construction Topological Structure. This construction focuses on practical application, covering the main campus of the school for Teaching, scientific research, office and Learning, life, exchange to provide practical and stable wireless network environment. The network topology is as follows. 


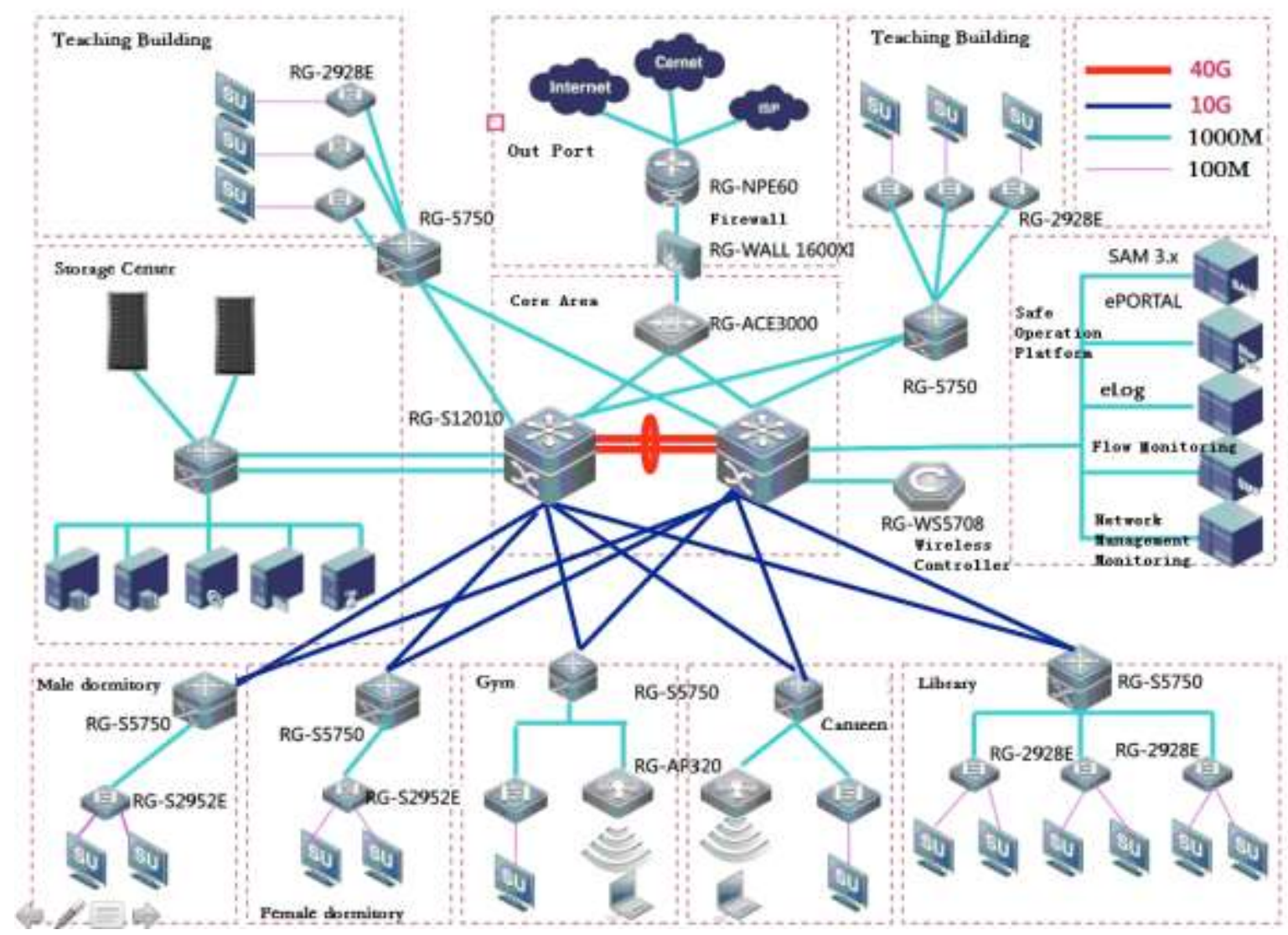

Figure 1. The Network Topology

Technical Advantages. The use of advanced wireless technology architecture: The wireless AP All the use of $\mathrm{H} 3 \mathrm{C}$ 802.11AC series products, indoor placement of AP using WA4320, indoor panel-type AP using WA4320H, outdoor AP Use of wa4320x, WA5320 and WA4320 series AP comply with the 802.11AC protocol standard and Support Wave2, can provide the whole gigabit access capability, is the same environment $802.11 \mathrm{n}$ products about 3 times times. It can effectively provide more high-performance mobile cloud access services from coverage, access density, and stability, and help users achieve the best TCO for their wireless networks (total cost of ownership/total costs of ownership).

Wireless network Management Advantages: This time the use of wireless management products for the entire wireless networks to provide self-learning, intelligent, real-time wireless resource management system, the specific advantages are as follows: real-time analysis of wireless resources, automatic distribution of wireless channels, wireless terminal viewing and roaming record audit, automatic set launch power, self-healing network, Scalable system, interference detection and avoidance, real-time monitoring network health, real-time load balancing.

\section{Wireless Network Solution Features}

Integrated Operation and Maintenance Management. This wireless network AP can support as a remote probe analysis of the sensor equipment, can be in the coverage of the Wi-Fi message capture and real-time mirroring to local analysis equipment, for network administrators to troubleshoot, optimization analysis. The remote probe analysis can not only mirror the work channel without convergence, but also can sample all channel polling and flexibly meet the requirements of wireless network monitoring and operation.

Wireless Intrusion Detection and Defense. Wireless networks are flexible, and infrastructure is invisible, making them more susceptible to unauthorized use than wired networks. For this kind of problem, the wireless network system provides the rogue equipment detection function, may detect 
the wireless intrusion, may clearly display the illegal access equipment, and carries on the information inquiry to it, joins the blacklist and launches the attack and so on the movement.

The mobile security defense modes supported by wired and wireless integrated switches are: Blacklist, whitelist, rogue defense, malformed message detection, illegal user's off-line, signature MAC layer attack detection and inversion based on preset upgrade (for example, Dos attack, flood attack, man-in-the-middle attack) and so on.With the wireless application console built in the mass of intelligent expert knowledge base, you can obtain a flexible wireless security policy to determine the basis for a clear source of illegal attacks (AP or terminal, etc.), the realization of visual physical location tracking monitoring and switch physical port removal.

Ealtime Spectrum Guard (RTSG) is the industry's most advanced professional monitoring solution for the spectrum status of wireless environments.RTSG's console fusion is deployed in the Network Intelligence Management Center, through Capwap management tunnels, communication and data acquisition with Sensor AP, 7x24-hour wireless environmental quality monitoring, wireless network capability Trend assessment, and non-license interference alarms. Through the graphical way, the active detection and identification of all $2.4 \mathrm{ghz} / 5 \mathrm{ghz}$ band of RF interference source (Wi-Fi or non-wi-Fi), can provide real-time FFT map, spectrum density map, spectral map, duty ratio map, event spectrum, channel power, interference power, etc. Can automatically identify the source of interference, determine the location of the problem of wireless devices, to ensure that the wireless network to perform the best performance. Combined with some intelligent reports, it can realize the storage, traceability and playback of the RF quality history record in the whole coverage area, automatically generate the trend of the customer, compliance and audit report.

For the different levels of user wireless environment supervision, the deployment of RTSG scheme can be flexibly implemented by local mode or monitor Mode. When working in local mode, it is possible to maintain normal user access and packet forwarding in the context of effective spectrum protection. As shown in the figure.2:

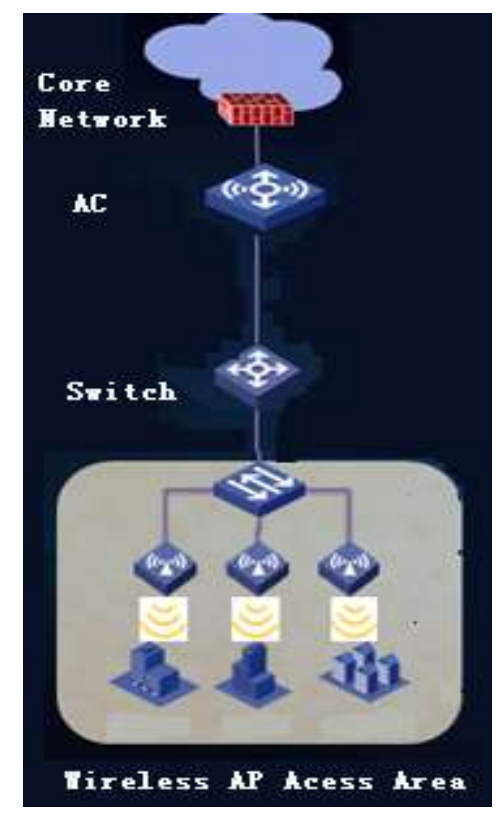

Figure 2. The RTSG Scheme

Spectrum navigation. The $5 \mathrm{G}$ frequency band has many channels, the channel interference is small, can give full play to $11 \mathrm{n}$ high rate advantage.

The Spectrum navigation function can automatically recognize single frequency and dual frequency network card, and guide dual-frequency network card to first access $5 \mathrm{G}$ channel. 


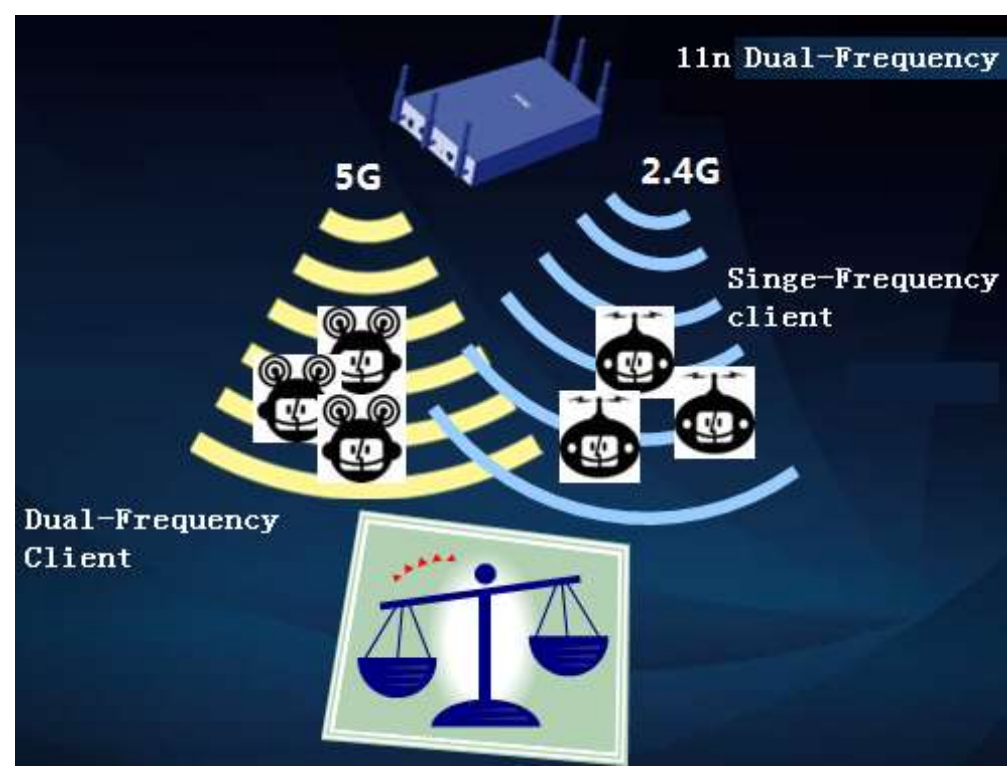

Figure 3. Spectrum Navigation

\section{Summary}

This paper takes the wireless network construction project of Jilin Business and Technology College as the research object, constructs the network topology based on the above technology. launches the implementation construction according to the design plan.

\section{Acknowledgements}

The project was supported by the Humanities and Social Science Research Project of Ministry of Educational (201702066013).

\section{References}

[1] Information on http://www.xjtu.edu.cn/xxfw/xywljs.htm.

[2] M.C.Zhu: Netwrok Planning and Optimization Technology (People's Postal Press Publications, China 2018).

[3] Information on https://wenku.baidu.com/view/a4fb0663700abb68a882fb9c.html?from=search.

[4] H.Li: A practical course in network planning and design (People's Postal Press Publications, China 2018).

[5] Z.R.Wan: Network Planning and System Integration (Beijing University of Aeronautics and Astronautics Press, China 2016).

[6] Information on https://wenku.baidu.com/view/7d0560e3376baf1ffd4fada0.html?from=search.

[7] Information on https://wenku.baidu.com/view/36c1334591c69ec3d5bbfd0a79563c1ec5dad7ec.html.

[8] Information on https://blog.csdn.net/mico_cmm/article/details/54176427.

[9]D.B.Zeng: Network Planning and Optimization (Beijing Polytechnic University Press, China 2018).

[10] M.L.Yao: CDMA2000 Network Planning and Optimization (Chemical Industry Press, China 2018). 